\title{
Best practice of Rendering CDA in a Cross Enterprise
}

\section{Document Sharing Environment}

\author{
Stefan Sabutsch ${ }^{1,2}$, Peter Seifter ${ }^{1,3}$ \\ ${ }^{1} \mathrm{HL} 7$ Austria, Austria \\ ${ }^{2}$ ELGA GmbH, Austria \\ ${ }^{3} \mathrm{FH}$ Joanneum $\mathrm{GmbH}$, University of Applied Sciences, Austria
}

\begin{abstract}
While much has been written about the clinical document architecture and the challenges on semantic interoperability, the modelling of content and the implementation, little attention has been paid to the representation of the medical payload in CDA documents and its implications on usability and accessibility.

The authors compare different methods to display CDA documents in a cross enterprise environment on the example of the Austrian national patient health record system. Strategies and decisions as well as technological approach and security implications are presented.
\end{abstract}

Finally, a combination of PDF/A-3 and attached CDA document is proposed as a best practice method for future implementations.

\section{Keywords}

Clinical Document Architecture; Rendering; XSLT Stylesheet; User-centric design; Eye tracking; Formatting Objects Processor; PDF/A-3 formatter

\section{Correspondence to:}

\section{Stefan Sabutsch}

HL7 Austria

Address: Eggenberger Allee 11, 8020 Graz, Austria

E-mail: stefan.sabutsch@hl7.at

\section{EJBI 2016; 12(1):en70-en76}

received: April 19, 2016

accepted: May 3, 2016

published: May 20, 2016

\section{Introduction}

One of the most important requirements of the CDA standard is that a CDA document has to be humanreadable via any common standard web browser. The clinical content of a CDA document is encoded via XML, formatting is possible with a reduced set of a HTML-like markup language [6. A web browser will show the human readable part of CDA (the so called narrative block) without any formatting and line breaks. The CDA document can be displayed as formatted HTML document if a "stylesheet" is used which enables an XSL Transformation (XSLT). XSLT stands for XSL Transformations, which converts the XML input in HTML output while the original input file remains unchanged. A standard web browser may act as an XSLT processor and as a renderer for the result HTML output, if the document is associated with an XML stylesheet. The name and path to the stylesheet may be indicated in the XML-header of the CDA file, so that the web browser can render the document automatically.

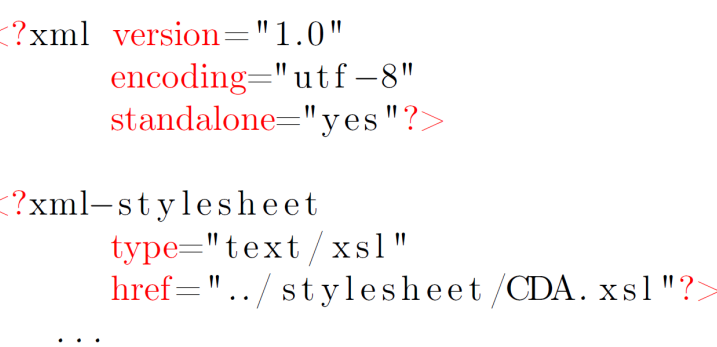

Health Level Seven International issued CDA Rel. 2 with a basic XSLT stylesheet (named CDA.xsl). Every standard conformant CDA document shall be displayed in a technically correct way with this instruction set, but only part of the administrative data in the CDA header is rendered. One of the main characteristics of CDA is, that it does not mandate a specific stylesheet to be used. Therefore, many different views on one document are possible and all CDA documents may be viewed with one stylesheet. A stylesheet must not add relevant information not present in the CDA document. If an additional different stylesheet were used, this information would be 
lost. A stylesheet must not be based on the machine readable content of the CDA document either as using the entries as a source for the stylesheet may lead to misinterpretation of the content 10.

However, the creator of a CDA document cannot be certain on how the document will be rendered by other recipients. An electronic signature of a CDA document is also possible and limited to the XML content unless no additional mechanisms are used [1. It is interesting to note that there exist only a handful of publications dealing in detail with the presentation of CDA documents [2, 11, 3].

The same is true for C-CDA that is used in the USA. As "many clinicians are frustrated with the usability of C-CDA", HL7 International and the Office of the National Coordinator for Health Information Technology (ONC) launched a "rendering tool challenge" for C-CDA in 2016 [4.

\subsection{ELGA - National health record system}

ELGA, the Austrian national patient health record ${ }^{1}$ was put in operation in December 2015. ELGA allows a cross domain exchange of health data between all authorized Austrian health care providers via IHE XDS/XCA profiles. In the first phase, four distinct document classes - physician and nurse discharge letters, laboratory and radiology reports - can be shared. The format for the documents is restricted to CDA Rel. 2. All files are required by law to conform to the nationally harmonized HL7 CDA implementation guidelines for proper use in ELGA. ELGA provides secure and reliable access to health data for any patient visiting a Health Care Provider (HCP). The data may be imported into the local electronic health record system (EHRS, e.g. general health practitioner or hospital clinical information system) where documents must be rendered. This implies that every EHRS has to deal with displaying ELGA documents, thus entailing the opportunity to adjust the appearance to the local graphical user interface and corporate design.

To facilitate the use of CDA documents for health IT system vendors, it was decided to provide stylesheets by ELGA. It quickly became apparent that the expectations of clinicians could not be met with the original stylesheet issued with the standard CDA Rel. 2. Consequently the stylesheet for the four document classes should be tailored to the specific requirements of the Austrian clinicians and should meet their demands for usability and accessibility. On the other hand it is allowed to implement any other type of displaying CDA documents in specific information systems.

At the beginning of the project methods to display CDA documents in a distributed multi-stakeholder environment on different IT systems were evaluated.

\section{User-centered design approach}

The deliverables of the different ELGA working groups from the years 2008-2015 have been adopted for the design of the stylesheet. The working groups consist of public, relevant stakeholders who were invited by the ELGA $\mathrm{GmbH}$ to send delegates. For the four different document classes "Physician Discharge Letter", "Nurse "Discharge Letter", "Laboratory Report" and "Radiology Report" separate working groups were appointed to guarantee transparent and consensus-based decisions. Experts of the working groups were interviewed to determine which administrative data is important in their daily work routine and must be visible "at first sight" and what information can be made visible by scrolling down and up and clicking onto the document. The individual experts generated a recommendation about the relevance, sequence and positioning of the administrative data.

On the basis of this recommendation a team of usability experts was able to draw up a vertical design prototype which was submitted to the Expert Group for approval. The usability of the draft was tested with test persons in a usability laboratory. Video-based eye trackers were used to get a better understanding of detecting the most relevant information, to increase operability and to avoid high complexity or distraction. Additionally, it was verified whether specific content could be realized correctly and interpreted by individual subjects. With the findings from the usability testing, the draft design prototype was revised and was used as a template for the implementation of the stylesheet. The ELGA reference stylesheet itself was implemented in software by external software companies.

\section{Transformation of CDA documents in a multi- stakeholder environment}

First, methods to display CDA documents in a distributed multi-stakeholder environment with different ITsystems were evaluated as to their feasibility and security. The development of a proprietary CDA viewer application for a rendering documents without the use of a web browser was not considered. Through literature search and analysis of state-of-the-art procedures we figured out five approaches to transform CDA documents:

\section{Global stylesheet (available at a central location)}

Every CDA document defines an absolute path to the corresponding stylesheet with public access via internet. The advantage of this method is that only a web browser with internet connection is necessary to represent a CDA document. At the same time this method includes a significant security risk because it enables the execution of codes. Unfortu-

\footnotetext{
${ }^{1}$ http://www.elga.gv.at
} 
nately, this method violates the same-origin policy in the web application security model, and thus ultimately represents a cross-site scripting, which is blocked in standard web browsers by default for security reasons. A reconfiguration of all browsers in the environment of ELGA is hardly feasible. Standard web browsers are blocking cross site scripting for security reasons. This option would require changing all security levels of all browsers involved which would be a task for service desks.

2. Local stylesheet (and providing a generic stylesheet) The transformation process is provided via a local stylesheet. Every CDA document includes a relative path to a reference stylesheet. If the file is defined without a path, then the stylesheet has to be in the same folder as the CDA document. A generic stylesheet could be available for download and the user has to make sure that this (or another) stylesheet matches the local path relative to the CDA document.

As long as the reference stylesheet can be downloaded from a trusted source in a secure manner, this method has the disadvantage that the distribution of new versions of the stylesheet can only be carried out by implementing organizational measures. The source of the stylesheet cannot be sure how the document is presented at the recipient.

3. Packaging CDA documents with a stylesheet

Another method is that a CDA document and the stylesheet will be delivered as one bundled package. As long as that CDA document is displayed with the attached stylesheet, the document is rendered as intended by the sender. The advantage of this method would be that for every document a customized style sheet can be provided. A disadvantage, however, is that the recipient eventually has to deal with a variety of stylesheets.

\section{Self Displaying CDA}

A (CSS) stylesheet can be embedded in the CDA document in a way that the file can be rendered without an external stylesheet [5. This solution could solve all problems as the rendering would happen automatically. HL7 International launched a project in 2010 to create a CSS stylesheet that would enable CDA documents to be displayed in a web browser without transformation. The main idea was to include a CSS stylesheet within a CDA document to enable the displaying of the content without the use of additional resources. It did not interfere with the ability to preferably display a CDA document with a locally defined stylesheet. However, the task could not be solved due to some technical problems, for instance embedded content could not be displayed with CSS. Thus, the project was withdrawn in 2012 without relevant results [8].

\section{PDF/A-3 with embedded CDA}

This method delivers the document as a PDF file and embeds the CDA file into the PDF file. The adopted standard PDF/A-3 (ISO 19005-3) which is based on PDF 1.7 (ISO 32000-1: 2008) has supported this variant since 2012 [9]. PDF is widely accepted as a standard document in the world of web. PDF documents can be displayed reliably, archived and viewed, printed and electronically signed in an easy way. Viewers are available free of charge. Therefore PDF is widely accepted as a standard document in the world of web. Major browsers (e.g. Firefox, Chrome) can show PDF files directly, and for other browsers (e.g. Safari) plugins usually exist. Semantic interoperability can be supplemented by embedding CDA documents in PDF files. This method has already been proposed on the website of HL7 Germany [7.

\section{Implementation of a CDA reference style sheet for ELGA}

The decision in favor of the preferred solution "PDF/A-3 with embedded CDA" was taken in 2011. At that time, PDF/A-3 was not yet available, and the "Self displaying CDA" was still far away from a sufficiently stable and functional implementation. The idea of a central stylesheet was dropped because of the security problems. Packaging of CDA documents with stylesheets appeared to be too complex and did not fit into the strict XDSbased architecture of ELGA. Therefore it was decided to publish an ELGA stylesheet as a template for further development.

At the beginning it was discussed whether the definition of a customized stylesheet for each document class in order to present the specific content is the best approach. For example, laboratory findings heavily differ in their tabular structure from other text-based documents such as discharge letters. However, it was decided that for all the documents a generic stylesheet should be used to ensure a uniform appearance of all CDA documents. Additionally with a distinctive design (recognition factor) a fast orientation within the structured document can be ensured.

Built on the results of the working groups the following design decisions were taken:

- Only the narrative block of a CDA document is rendered (no level 2 and 3 content)

- A table of contents of the most important metadata and sections will be prefixed

- Important meta data precede the clinical content, less important information can be folded or be placed at the end of the document

- Title, date, version 
- Patient name, date of birth, social security number, procurator/trustee/legal guardian

- Encounter

- Author, receiver (if indicated)

- Other metadata is put at the end or can be unfolded

- No sorting, ordering or filter functionality in the clinical content to avoid the risk to misinterpret the information of the document by any sequence of sections In practice there is no guarantee that the section texts are filled in correctly and associated (e.g. allergies or diagnoses are in the "wrong" sections)

- Embedded multimedia files are allowed (ELGA accepts only a few multimedia formats: PDF, JPG, PNG, GIF, MPEG, MP3)

- Simple print function (similar to the on-screen display)

- Specific markups - in everyday clinical practice, it is helpful to highlight texts with colors. Pathological laboratory values, for example, are not typed in bold only, but also use red color to distinguish them from normal values. Color highlighting may be made only in blue (red is not perceived by many people) exclusively. Blue can be seen by color-blind people as grey.

- Typography: Usage of a legible on-screen sans serif font (Arial, sans-serif) that is supported by all major browsers and font size of text body with $100 \%$ - so the text is displayed in the size defined by the user.

- Limited line width - on modern monitors the browser window can be very broad, the eye finds it harder to jump back to the next line in wide rows harder. Therefore, the line width was fixed to 900 pixels.

- Ragged alignment, bigger line spacing (compared to printed documents)

- Section text is indented after the heading. Jumping between paragraphs is easier for the eye.

- Tables are always represented in the same width as the body text.

- Column width of tables: CDA documents allow the use of tables, but do not allow the user to define the column width. Therefore the project-specific markups, which have been developed for ELGA, can be used in reference stylesheet.

- Table rows with alternating background color

- Interactive elements (e.g. to fold out) always have a hover function (i.e. change the appearance when the mouse is being passed over it)
- Exclusive use of a small set of colors according to accessibility criteria. Colors have to be clearly visible and distinguishable in printed form: black, grey, blue, yellow, bright yellow

- Display embedded images and other multimedia files automatically

- Translation of coded values and "NullFlavors" in the metadata through translation tables inside the stylesheet regardless of the display name (e.g. translate LOINC code to text, translate "NullFlavors" for birth date UNK to "unknown date of birth")

- Usage of HTML 4.0 for strictly conforming XHTML 1.0 documents and UTF-8

- Stability against non-conforming content in the narrative block

- Robustness against maliciously composed documents

Of course some limitations have to be accepted:

- While the default browser is capable of directly displaying Base64 embedded JPG and GIF images, other media types can only be displayed if they have been previously decoded. This functionality cannot be implemented by an XSLT stylesheet. A Base64 decoder servlet must be available in the local working environment. ELGA provides a base servlet available for download. If the decoder is missing, an appropriate error message is displayed in the output document.

- CDA with "unstructured body" can only show the information of the header. The PDF file has to be opened by the user with one mouse click.

Figure 1 shows an example CDA document using the reference stylesheet.

\subsection{Transformation process for printing and long-term archiving}

Due to the different possibilities of monitor screens and paper the requirements to print CDA documents differ from the screen-oriented representation:

- Printable representation similar to the HTML output (as much as possible)

- Headers and footers with serial numbering

- Printing document and PDF Attachments

- Scaling the graphics and page limitation

- Placeholder for multimedia files (audio, video) with a notice

- File format supporting long-term archiving (PDF/A) 
- Tagged PDF (requirement by accessibility)

- Option to show the interactive HTML "folded" information

- Embedding of the original CDA document (PDF/A3)

- Hyphenation, paragraph settings to automatically prevent widows and orphans

These requirements are made possible by the use of XSL-FO (Extensible Stylesheet Language - Formatting Objects) and a corresponding FOP (Formatting Objects Processor) from the Apache project. XSL-FO was designed for paged media and the concept of pages is an integral part of XSL-FO structure. It is capable of a great deal of expressiveness. Tables, lists, side floats, and a variety of other features are available. 12

The software executable, called CDA2PDF converter, cannot be directly executed in the browser, therefore a servlet container is required.

The conversion of a "ELGA" CDA document to PDF format looks similar to the HTML browser-based document (see figure 2). Noteworthy is the page footer with the additional file name and the information about the creation of the PDF file placed on the left side. Not visible are the folded information blocks, which are placed on the last page, because they are hidden behind interactive HTML elements.

\section{Conclusion and Discussion}

\subsection{Security Considerations}

Although the injection of malicious code (e.g. Javascript) can be inhibited in the stylesheet, the multimedia content is also a potential security risk. This cannot be blocked by the stylesheet itself. If the media type (PDF, MPEG) is approved in principle the content cannot be checked by the stylesheet. In the ELGA environment firewalls were built that block both malicious code in multimedia attachments as well as in not well-formed or non-conforming XML data. In addition a schema validation and validation of correct PDF/A file format in the attachments will be carried out in the near future.

\subsection{Electronic Signature}

In a PDF/A file content and presentation are not separated, so a PDF/A document can easily be signed electronically. In comparison, the electronic signature of a CDA document is fundamentally flawed and is not provided in the CDA standard. This kind of signature would also include the representation (i.e. the stylesheet). If only the CDA document is signed, and not the XSLT stylesheet, an inconsistent presentation of CDA document may occur. The principle of implementing the electronic

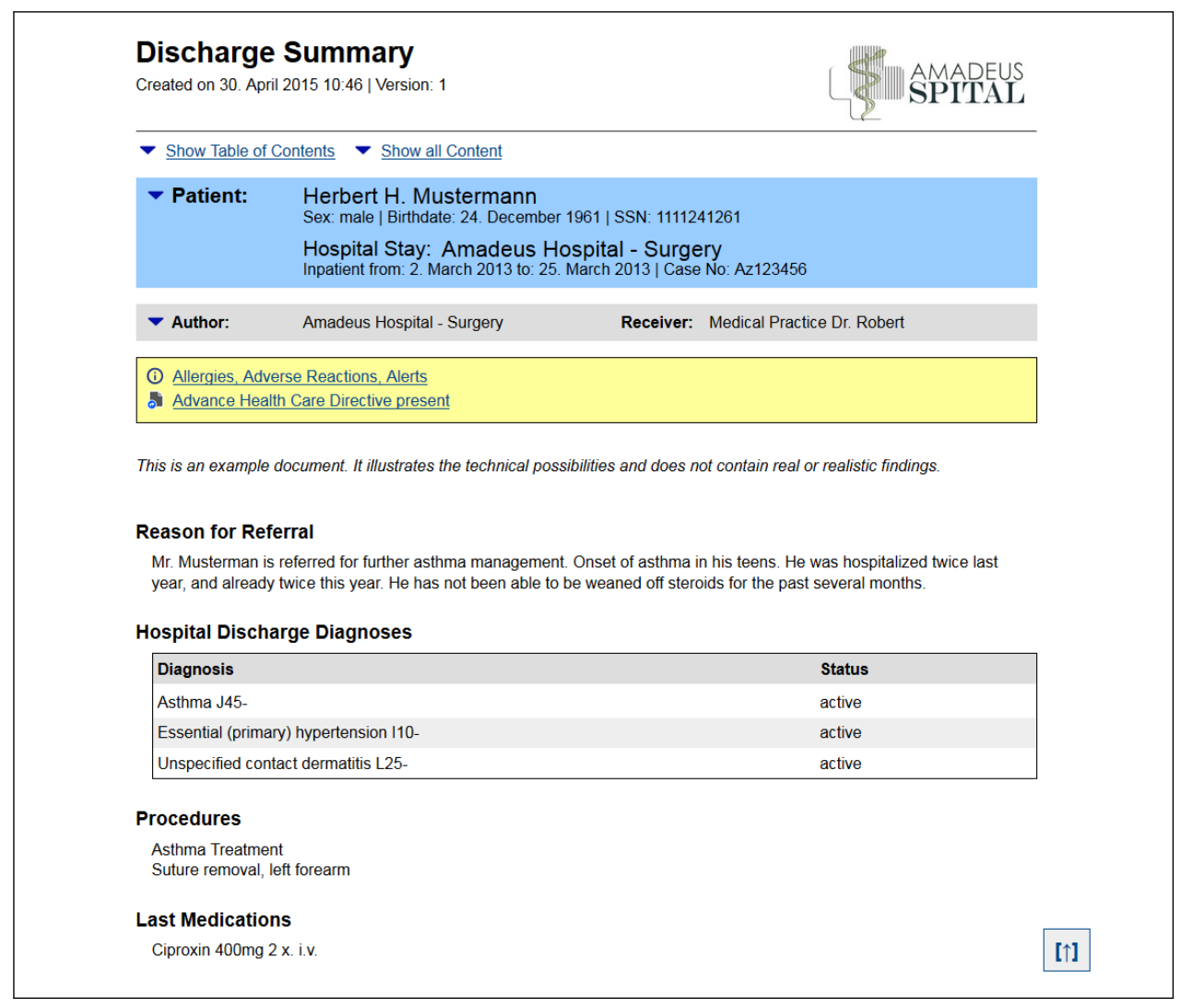

Figure 1: Reference stylesheet 
signature for CDA documents should include the clinical data and also the representation part.

In the "ELGA ecosystem" an electronic signature for CDA documents is not intended and is not necessary. Although an electronic signature can be applied to the original documents in the individual EHRS, the exchange format CDA only contains an indication that the original document has been signed. However, the ELGA infrastructure ensures that only identified and authorized HCP can register documents. At the same time the immutability ("file fixity") is ensured by hash values (document and registry entry) in the document registry.

\subsection{Best practice solution}

From these considerations and the experience gained from the ELGA project we propose a best practice solution for future similar projects.

PDF/A-3 files including an attached CDA document should be used for the exchange of documents. The PDF/A-3 files shall be generated via a common XSL-FO converter from a CDA document as input file. The resulting PDF file may easily be electronically signed, exchanged, displayed and archived. By attaching the origi-

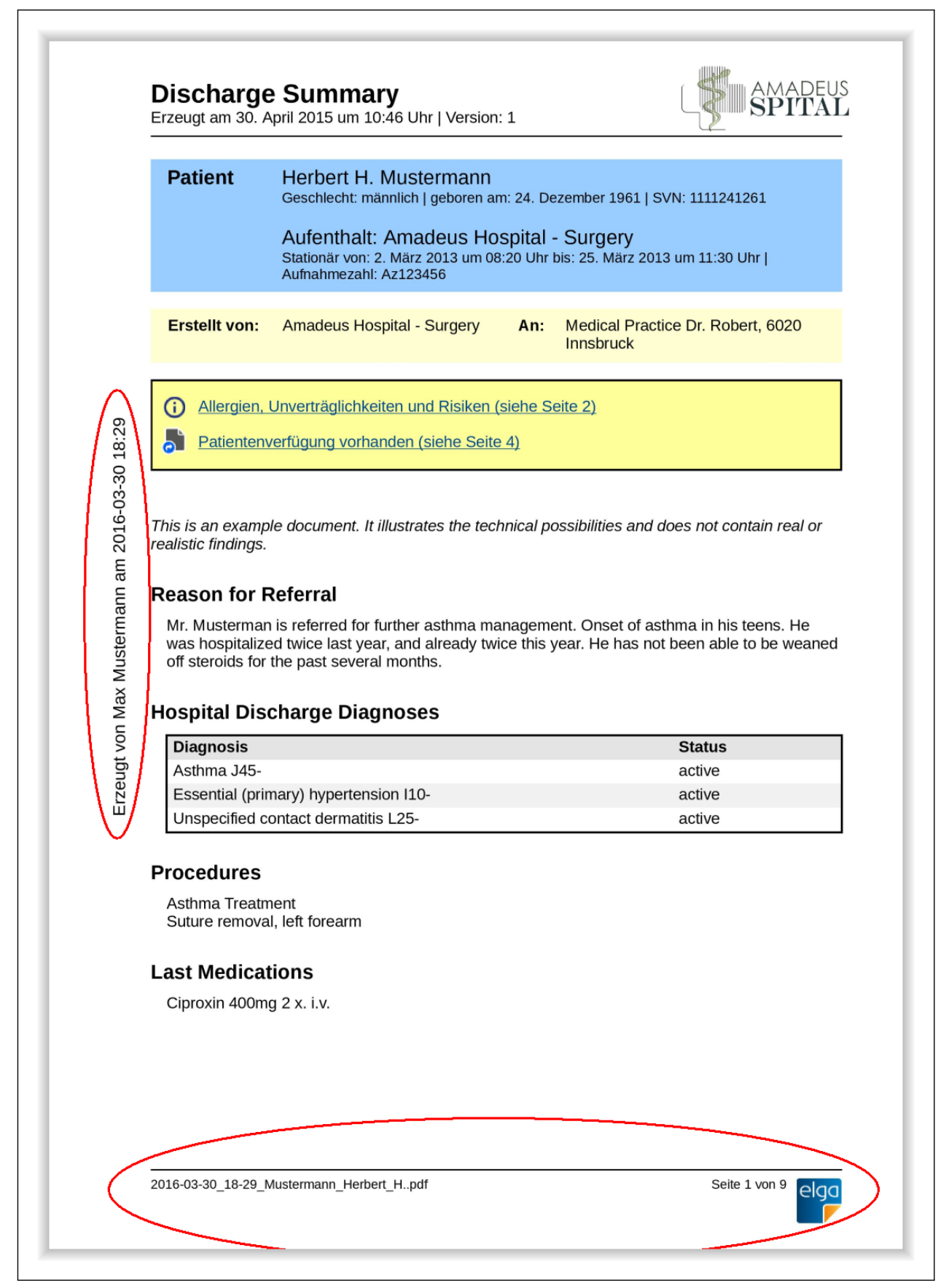

Figure 2: CDA document in PDF format 
nal CDA document all features of the CDA standard, in particular semantic interoperability, will be preserved.

CDA is essential from the point of view of semantic interoperability. CDA documents contain the relevant information for the document management and potentially machine-readable clinical content. PDF/A offers clear advantages in terms of immutability of the representation and long-term archiving, widespread tools for the representation and an electronic signature. PDF/A-3 files including a CDA document as an attachment combines the best of both worlds.

To ensure that PDF/A-3 files are generated correctly with a uniform appearance, an appropriate converter CDA2PDF can be provided which generates a corresponding pdf output file from a CDA document. The feasibility of the converter has been proven in ELGA and the converter uses the ELGA web portal to produce clinical documents for all Austrian citizens.

\section{Acknowledgements}

The authors are indebted to their colleagues from SDOs such as HL7 for open minded and kind cooperation and valuable support.

\section{References}

[1] Ärztekammer Nordrhein Elektronischer Arztbrief (XMLSignaturspezifikation, Schema, Beispiele) [Internet]. 2016 [cited 2016 Feb 03]; Available from:

http://www.aekno.de/page.asp?pageID $=6684$

[2] Bludau HB., Wolff A., Hochlehnert AJ. Presenting XML-based medical discharge letters according to CDA. Methods Inf Med.
$2003 ; 42: 552-6$

[3] Burgsteiner H., Kleinoscheg G., Hussa M. An CDA implementation guide about cardiopulmonary exercise testing (CPET) results. European Journal for Biomedical Informatics. $2012 ; 8(4): 16-23$

[4] Health Level Seven, Inc. C-CDA@C Rendering Tool Challenge [Internet]. 2016 [cited 2016 Feb 03]; Available from: http://www.hl7.org/events/toolingchallenge.cfm

[5] W3C Cascading Style Sheets Level 2 Revision 1 (CSS 2.1) Specification [Internet]. 2014 [cited 2016 Feb 03]; Available from: https://www.w3.org/TR/CSS21/

[6] Health Level Seven, Inc. (2005) HL7 Clinical Document Architecture, Release 2.0 HL7 (C) Version 3 Standard.

[7] Health Level Seven, Deutschland e.V. IG:CDA und PDF/A3 [Internet]. 2016 [cited 2016 Feb 03]; Available from: http://wiki.hl7.de/index.php/IG:CDA_und_PDF/A3

[8] Health Level Seven, Inc. C-CDA(C) Self Displaying CDA [Internet]. 2010 [cited 2016 Feb 04]; Available from: http://wiki.hl7.org/index.php?title=Self_Displaying_CDA

[9] ISO 19005-3:2012 Document management - Electronic document file format for long-term preservation - Part 3: Use of ISO 32000-1 with support for embedded files (PDF/A-3). 2012

[10] Spronk R, Grieve G Common issues found in implementations of the HL7 Clinical Document Architecture (CDA) [Internet]. 2016 [cited 2016 Feb 03]; Available from: http://www.ringholm.com/docs/03020_en_HL7_CDA common issues_error.htm

[11] Wolf KH., Schirmer S., Marschollek M., Haux R. Representing Sensor Data Using the HL7 CDA Personal Healthcare Monitoring Report Draft. Stud Health Technol Inform. 2009;150:480-4.

[12] W3C Extensible Stylesheet Language (XSL) Version 1.1 [Internet]. 2006 [cited 2016 Feb 03]; Available from: https://www.w3.org/TR/xsl11/ 